\title{
Adaptive Variable Structure Control for Mismatched Uncertain Systems
}

\author{
Bach Hoang Dinh ${ }^{1}$, Van Van Huynh ${ }^{1}$ \\ ${ }^{1}$ Department of Power Systems, Faculty of Electrical \& Electronics Engineering, Ton Duc Thang University, Ho Chi \\ Minh City, Vietnam \\ Correspondence: Van Van Huynh, Department of Power Systems, Faculty of Electrical \& Electronics Engineering, Ton \\ Duc Thang University, Ho Chi Minh City, Vietnam
}

\author{
Received: April 19, 2016 Accepted: May 3, $2016 \quad$ Online Published: July 12, 2016 \\ doi:10.11114/set.v3i1.1564 URL: http://dx.doi.org/10.11114/set.v3i1.1564
}

\begin{abstract}
This paper proposes an adaptive variable structure control (VSC) for a class of mismatched uncertain systems with unknown disturbances. First, a necessary and sufficient condition in terms of linear matrix inequalities is proposed to guarantee the system in sliding mode is asymptotically stable. Second, an adaptive output feedback variable structure controller is designed to force the system states reach the sliding surface and stay on it thereafter. Finally, the advantages and effectiveness of the proposed approaches are demonstrated via a numerical example.
\end{abstract}

Keywords: adaptive output feedback control, Variable structure control, mismatched uncertain systems

\section{Introduction}

Variable structure control (VSC) has emerged as a method capable of use in given robust control systems (Kwan, 1996; Zak \& Hui, 1993). The conventional VSC design can be divided into two phases. The first phase is the reaching phase, in which a switching control law is given so that the system trajectory can be trapped on a switching surface and remain on it thereafter. The second one is the sliding phase. The switching surface was determined in such a way that the system dynamics can have good performance in the sliding mode (Tsai, Mai, \& Shyu, 2006). The main advantages of VSC are fast response and strong robustness with respect to uncertainties and disturbances. However, in terms of implementing variable structure control scheme on practical systems of industrial relevance, the assumption of all the system states availability, including the initial conditions, is a usually conservative and imposes high set-up cost due to high number of sensors. In this situation, there are two approaches in designing the output feedback variable structure controllers. One is to use state observers to provide an estimate of the unmeasured states. The other is to utilize the output-based controllers, such as static gain and dynamic compensator. Thus, the design of asymptotic observers and dynamic compensators is very important and has been established (Diong \& Medanic, 1992; Emelyanov, Korovinj, Nersisyan \& Nisenzov, 1990; Esfandiari \& Khalil, 1992). However, the direct output feedback design in variable structure system (VSS) is worth investigating (Shyu, Tsai, \& Lai, 2001; Kwan, 2001; Choi, 1998; Silva, Edwards \& Spurgeon, 2009; Nunes, Hsu \& Lizarralde, 2009; Hao \& Yang, 2013; Zhang \&Zheng, 2014). The work by Shyu, Tsai, $\&$ Lai 2001) proposed an output feedback controller for matched uncertain systems. Another direct output feedback design was also proposed by Kwan (2001), which discussed the controllers' design for uncertain systems with matching conditions. In Choi's study (1998), the problem of designing a linear sliding surface was proposed for a class of uncertain systems with mismatched uncertainties. The works by Silva, Edwards \& Spurgeon (2009) proposed a sliding mode output feedback controllers for plants with matched and mismatched uncertainties. By using a hybrid compensation scheme, Nunes, Hsu \& Lizarralde (2009) presented a new variable structure control law to solve a longstanding problem of global exact output tracking for uncertain linear plants. The study by Hao \& Yang (2013) proposed the robust fault-tolerant control problem for uncertain linear systems via sliding mode output feedback. By Zhang \&Zheng (2014), the problem of designing adaptive output feedback variable structure controllers was developed for a class of linear systems with matched external disturbance. In order to handle a larger class of mismatched uncertainties, the authors of Ginoya, Shendge, \& Phadke (2014) presented a new sliding mode observer for general $n$th order systems with mismatched uncertainties. As a result, the closed-loop of mismatch uncertain systems driven by the proposed output feedback variable structure control scheme is asymptotical stable. However, it should be noted that most of the existing results for mismatch uncertain systems are required that the disturbances are bounded by a known 
upper bounding function. In this paper, an adaptive variable structure control scheme is proposed for a class of systems where disturbances are bounded by an unknown function.

\section{Statement of the Problem}

Let the system to be controlled be represented by the following differential equation:

$$
\begin{aligned}
& \dot{x}=[A+\Delta A(x, t)] x+B[u+g(x, t)] \\
& y=C x
\end{aligned}
$$

where $x \in R^{n}$ is the state vector, $u \in R^{m}$ is the control input, $y \in R^{p}$ is the output and $m \leq p<n$. The matrices $A, B$ and $C$ are constant matrices with appropriate dimensions. The term $\triangle A$ represents the mismatched uncertainty of the plant which the matching condition is not satisfied and $g(x, t)$ is the disturbance input.

For the system, the following assumptions are made

Assumption 1: $\Delta A(x, t)$ is of the form

$$
\Delta A(x, t)=D F(x, t) E
$$

where $F(x, t)$ is unknown but bounded as $\|F(x, t)\| \leq 1$ for all $(x, t) \in R^{n} \times R$, and $D$ and $E$ are known matrices of appropriate dimensions.

Assumption 2: The exogenous disturbance $g(t, x)$ is assumed to be bounded by a r-order polynomial of the norm of the output variables

$$
\|g(x, t)\| \leq a_{1}+a_{2}\|x\|\|y(t)\|+a_{3}\|x\|\|y(t)\|^{2}+\ldots+a_{r}\|x\|\|y(t)\|^{r}
$$

where the scalars $a_{1}, a_{2}, a_{3}, \ldots, a_{r}$ are unknown bounds, which are not easily obtained due to the complicated structure of the uncertainties in practical control systems.

Assumption 3: The matrices $\mathrm{B}$ and $\mathrm{C}$ are full rank, and $\operatorname{rank}(C B)=m$.

Under assumption 3, it follows from paper (Yan, Spurgeon, \& Edwards, 2012) that there exists a coordinate transformation $z=T x$ such that the system (1) has following regular form.

$$
\begin{gathered}
\dot{z}(t)=\left(\left[\begin{array}{cc}
A_{1} & A_{2} \\
A_{3} & A_{4}
\end{array}\right]+\left[\begin{array}{l}
D_{1} \\
D_{2}
\end{array}\right] F\left[\begin{array}{ll}
E_{1} & E_{2}
\end{array}\right]\right) z(t)+\left[\begin{array}{l}
0 \\
B_{2}
\end{array}\right][u+g(z, t)] \\
y(t)=\left[\begin{array}{ll}
0 & C_{2}
\end{array}\right] z(t)
\end{gathered}
$$

where $T A T^{-1}=\left[\begin{array}{cc}A_{1} & A_{2} \\ A_{3} & A_{4}\end{array}\right], T D F E T^{-1}=\left[\begin{array}{c}D_{1} \\ D_{2}\end{array}\right] F\left[\begin{array}{ll}E_{1} & E_{2}\end{array}\right], T B=\left[\begin{array}{l}0 \\ B_{2}\end{array}\right]$ and $C T^{-1}=\left[\begin{array}{ll}0 & C_{2}\end{array}\right]$. The matrices $B_{2} \in R^{m \times m}$ and $C_{2} \in R^{p \times p}$ are non-singular.

Let $z(t)=\left[\begin{array}{lll}z_{1}^{T}(t) & \mathrm{z}_{2}^{T}(t)\end{array}\right]^{T}$ then the equation of (2) can be rewritten as

$$
\begin{gathered}
\dot{z}_{1}(t)=\left(A_{1}+D_{1} F E_{1}\right) z_{1}(t)+\left(A_{2}+D_{1} F E_{2}\right) z_{2}(t) \\
\dot{z}_{2}(t)=\left(A_{3}+D_{2} F E_{1}\right) z_{1}(t)+\left(A_{4}+D_{2} F E_{2}\right) z_{2}(t)+[u+g(z, t)]
\end{gathered}
$$


The output sliding surface can be defined as follows:

$$
\begin{aligned}
\sigma(y(t))= & K y(t)=K C x(t)=K\left[\begin{array}{ll}
0 & \mathrm{C}_{2}
\end{array}\right] z(t) \\
& =K_{2} z_{2}(t)=0
\end{aligned}
$$

where $K \in R^{m \times p}$ are obtained from the algorithm given in (Edwards and Spurgeon, 1998) and $K_{2} \in R^{m \times m}$ is non-singular.

In sliding mode $\sigma(y(t))=0$ and $\dot{\sigma}(y(t))=0$, we have $z_{2}(t)=0$. Then, from equations (4) and (6), the sliding mode dynamics of system (1) associated with the sliding surface $(6)$ is described by

$$
\dot{z}_{1}(t)=\left(A_{1}+D_{1} F E_{1}\right) z_{1}(t)
$$

In what follows, our attention will be focused on two importance steps. The first step, appropriate LMI stability conditions by the Lyapunov method are derived such that sliding motion (7) is asymptotically stable. The second step, an adaptive output feedback variable structure control scheme is designed such that the system states reach the sliding surface (6) in finite time and stay on it thereafter.

Before proceeding, we will need the following lemma

Lemma 1 (Choi, 1998): Let $X, Y$ and $F$ are real matrices of suitable dimension with $F^{T} F \leq I$ then, for any scalar $\varphi>0$, the following matrix inequality holds:

$$
X F Y+Y^{T} F^{T} X^{T} \leq \varphi^{-1} X X^{T}+\varphi Y^{T} Y .
$$

\section{Sliding Mode Stability Analysis}

In this section, the stability of the sliding motion is investigated. Let us begin with considering the following LMI:

$$
\left[\begin{array}{lcc}
A_{1}^{T} P+P A_{1} & P D_{1} & E_{1}^{T} \\
D_{1}^{T} P & -\varphi^{-1} I & 0 \\
E_{1} & 0 & -\varphi I
\end{array}\right]<0
$$

where $P \in R^{(n-m) \times(n-m)}$ is any positive matrix and the scalar $\varphi>0$. Then, the following theorem shows that sliding motion (7) is asymptotically stable.

Theorem 1: Suppose that LMI (8) has a solution $P>0$ and the scalar $\varphi>0$. The sliding surface is given by equation (6). Then, the sliding motion described in (7) is asymptotically stable.

Proof: Now we are going to prove theorem 1. First, let us define a Lyapunov function candidate as

$$
V=z_{1}^{T} P z_{1}
$$

where the positive-definite matrix $P$ is defined in (8). If we differentiate $V$ with respect to time combined with (7) then

$$
\dot{V}=\dot{z}_{1}^{T} P z_{1}+z_{1}^{T} P \dot{z}_{1}=z_{1}^{T}\left[\left(A_{1}+D_{1} F E_{1}\right)^{T} P+P\left(A_{1}+D_{1} F E_{1}\right)\right] z_{1}
$$

Using Lemma 1, we obtain that for the scalar $\varphi>0$

$$
\dot{V} \leq z_{1}^{T}\left(A_{1}^{T} P+P A_{1}+\varphi P D_{1} D_{1}^{T} P+\varphi^{-1} E_{1}^{T} E_{1}\right) z_{1}
$$

By the Schur complement, (8) is equivalent to

$$
A_{1}^{T} P+P A_{1}+\varphi P D_{1} D_{1}^{T} P+\varphi^{-1} E_{1}^{T} E_{1}<0 .
$$

After all, from equations (11) and (12), it is clearly that 


$$
\dot{V}<0 \text {. }
$$

The inequality (13) implies that if the LMI (8) is feasible then sliding motion (7) is asymptotically stable.

Remark 1: Theorem 1 provides the new existence condition of the sliding mode via LMI technique, which can be easily worked out using LMI Toolbox in Matlab.

\section{Reachability Analysis}

The objective in this section is to design a static output feedback sliding mode control such that system states are driven to the sliding surface (6). In order to satisfy the above aim, the modified variable structure controller is selected to be

$$
\left.u(t)=-B_{2}^{-1}\left[\left\|K\left[\begin{array}{ll}
0 & C_{2}
\end{array}\right] T A T^{-1}\right\|+\left\|K\left[\begin{array}{ll}
0 & C_{2}
\end{array}\right] T D\right\|\left\|E T^{-1}\right\|\right) \eta+\alpha+\zeta(t)\right] \frac{\sigma}{\|\sigma\|}
$$

where scalars $\alpha>0, \eta>0$ and the adaptive law is defined as:

$$
\zeta(t) \geq \sum_{i=1}^{r} \hat{a}_{i}(t) \eta\|\sigma\|\left\|B_{2}\right\|\left\|T^{-1}\right\|\|y\|^{i-1}
$$

where $\hat{a}_{i}(t)$ is the solution of the following equations

$$
\dot{\hat{a}}_{i}(t)=q_{i} \eta\|\sigma\|\left\|B_{2}\right\|\left\|T^{-1}\right\|\|y\|^{i-1}, i=1 \ldots r
$$

in which scalars $q_{i}>0, i=1 \ldots r$.

The following Theorem is established to discuss detail conditions that system states are driven to the sliding surface (6).

Theorem 2: Suppose that the LMI (8) has a solution $P>0$ and the sliding surface is given by equation (6). Consider the closed loop of system (2)-(3), then the control law (14) guarantees that the system state reaches the sliding surface in finite time for $\|z(t)\| \leq \eta$.

Proof: Let us consider the following Lyapunov function

$$
V=\frac{1}{2} \sigma^{T} \sigma+\frac{1}{2} \sum_{i=1}^{r} \frac{\tilde{a}_{i}^{2}(t)}{q_{i}}
$$

where $\tilde{a}_{i}(t)=a_{i}-\hat{a}_{i}(t)$, the scalar $q_{i}>0 \quad i=1, \ldots, r$. By differentiating $V$ with regard to time using (6) yields that

$$
\begin{aligned}
& \dot{V}=\sigma^{T} \dot{\sigma}-\sum_{i=1}^{r} \frac{\tilde{a}_{i}(t) \dot{\text { 染}(t)}}{q_{i}}=\sigma^{T} K \dot{y}(t)-\sum_{i=1}^{r} \frac{\tilde{a}_{i}(t) \dot{a}_{i}(t)}{q_{i}} \\
& =\sigma^{T} K\left[0 \quad C_{2}\right] \dot{z}(t)-\sum_{i=1}^{r} \frac{\tilde{a}_{i}(t) \dot{\hat{a}}_{i}(t)}{q_{i}} .
\end{aligned}
$$

Substituting equation (2) into equation (18), one can get

$$
\begin{aligned}
\dot{V}= & \sigma^{T} K\left[\begin{array}{ll}
0 & C_{2}
\end{array}\right]\left(T A T^{-1}+\operatorname{TDFET} T^{-1}\right) z(t)+\sigma^{T} B_{2}[u+g(z, t)]-\sum_{i=1}^{r} \frac{\tilde{a}_{i}(t) \dot{\hat{a}}_{i}(t)}{q_{i}} \\
\leq & \|\sigma\|\left(\left\|K\left[\begin{array}{ll}
0 & C_{2}
\end{array}\right] T A T^{-1}\right\|+\left\|K\left[\begin{array}{ll}
0 & C_{2}
\end{array}\right] T D\right\|\left\|E T^{-1}\right\|\right) \eta+\sigma^{T} B_{2} u(t) \\
& +\|\sigma\|\left\|B_{2}\right\|\|g(z, t)\|-\sum_{i=1}^{r} \frac{\tilde{a}_{i}(t) \dot{\hat{a}}_{i}(t)}{q_{i}} .
\end{aligned}
$$

From assumption 2 we have 


$$
\begin{aligned}
\dot{V} & \leq\|\sigma\|\left(\left\|K\left[\begin{array}{ll}
0 & C_{2}
\end{array}\right] T A T^{-1}\right\|+\left\|K\left[\begin{array}{ll}
0 & C_{2}
\end{array}\right] T D\right\|\left\|E T^{-1}\right\|\right)\|z\|+\sigma^{T} B_{2} u(t) \\
& +\sum_{i=1}^{r} a_{i}\|\sigma\|\left\|B_{2}\right\|\left\|T^{-1}\right\|\|z\|\|y\|^{i-1}-\sum_{i=1}^{r} \frac{\tilde{a}_{i}(t) \dot{\hat{a}}_{i}(t)}{q_{i}} \\
& \leq\|\sigma\|\left(\left\|K\left[\begin{array}{ll}
0 & C_{2}
\end{array}\right] T A T^{-1}\right\|+\left\|K\left[\begin{array}{ll}
0 & C_{2}
\end{array}\right] T D\right\|\left\|E T^{-1}\right\|\right) \eta+\sigma^{T} B_{2} u(t) \\
& +\sum_{i=1}^{r} a_{i} \eta\|\sigma\|\left\|B_{2}\right\|\left\|T^{-1}\right\|\|y\|^{i-1}-\sum_{i=1}^{r} \frac{\tilde{a}_{i}(t) \dot{\hat{a}}_{i}(t)}{q_{i}} .
\end{aligned}
$$

Substituting equations (14)-(16) into equation (20), it is clearly that

$$
\dot{V} \leq-\alpha-\xi(t)+\sum_{i=1}^{r} a_{i} \eta\|\sigma\|\left\|B_{2}\right\|\left\|T^{-1}\right\|\|y\|^{i-1}-\sum_{i=1}^{r} \tilde{a}_{i}(t) \eta\|\sigma\|\left\|B_{2}\right\|\left\|T^{-1}\right\|\|y\|^{i-1} .
$$

Clearly, we obtain

$$
\dot{V} \leq-\alpha<0
$$

Thus, the proof is completed.

Remark 2: From VSC theory, Theorems 1 and 2 together show that the sliding surface (6) with the output feedback variable structure controller (14) guarantee that: 1 ) at any initial value the system states reach the sliding surface and stay on its thereafter; and 2) the mismatched uncertain system (1) is asymptotically stable.

\section{Numerical Example}

In this section, we test the proposed adaptive output feedback controller on the mismatched uncertain system used by Silva, Edwards \& Spurgeon (2009)

$$
\begin{aligned}
\left.\dot{x}=\left(\begin{array}{rrr}
1 & 1 & -1 \\
1 & -1 & 0 \\
4 & 0 & 2
\end{array}\right]+\Delta A\right) x+\left[\begin{array}{l}
0 \\
0 \\
1
\end{array}\right](u+g) & \\
y & =\left[\begin{array}{lll}
0 & 1 & 0 \\
0 & 0 & 1
\end{array}\right] x
\end{aligned}
$$

where the mismatched uncertain is given as $\triangle A=D F E$ with $D=\left[\begin{array}{lll}1 & 1 & 1\end{array}\right]^{T}, \quad E=\left[\begin{array}{lll}1 & 1 & 1\end{array}\right]$ and $F=0.9 \sin (x) ;$ the disturbance is assumed to satisfy as $\| \xi(x, t))\|\leq 0.01+0.9\| y\|+0.9\| y \|^{2}$.

The coordinate transformation is given as $T=\left[\begin{array}{ccc}-1 & 0 & 0 \\ 0 & -1 & 0 \\ -5 & 0 & 1\end{array}\right]$. The matrices $B_{2}=1, C_{2}=\left[\begin{array}{rr}-1 & 0 \\ 0 & 1\end{array}\right]$ are non-singular and the matrix $A_{1}=\left[\begin{array}{cc}-4 & 1 \\ 1 & -1\end{array}\right]$ is stable. Using LMI approach which mentioned in (8), we can find the 
solution $P=\left[\begin{array}{ll}0.7651 & 0.7767 \\ 0.7767 & 3.0997\end{array}\right]$. The sliding matrices are given by $K=\left[\begin{array}{ll}1 & 1\end{array}\right]$ and from (6) the sliding surface is consider as

$$
\sigma(y, t)=\left[\begin{array}{ll}
1 & 1
\end{array}\right] y(t)=0
$$

Let $\alpha=30.8$ and $\eta=2$, then the control law is reachable from (14) which is

$$
u(t)=-[\zeta(t)+67.1] \frac{\sigma}{\|\sigma\|}
$$

where $\zeta(t) \geq 10.3$ 海 $(t)\|\sigma\|+10.3 a_{2}(t)\|\sigma\|\left\|y_{1}\right\|+10.3 a_{3}(t)\|\sigma\|\left\|y_{1}\right\|^{2}, \quad$ and $\quad \hat{a}_{1}(t), \hat{a}_{2}(t)$ and $\hat{a}_{3}(t)$ satisfy the following equations

$$
\begin{gathered}
\text { 染 }(t)=0.1\|\sigma\|, a_{1}(0)=0.02, \\
\text { 泈 }(t)=0.1\|\sigma\|\|y\|, a_{2}(0)=0.01
\end{gathered}
$$

and

$$
\text { 深 }(t)=0.1\|\sigma\|\|y\|^{2}, a_{3}(0)=0.02
$$

respectively.

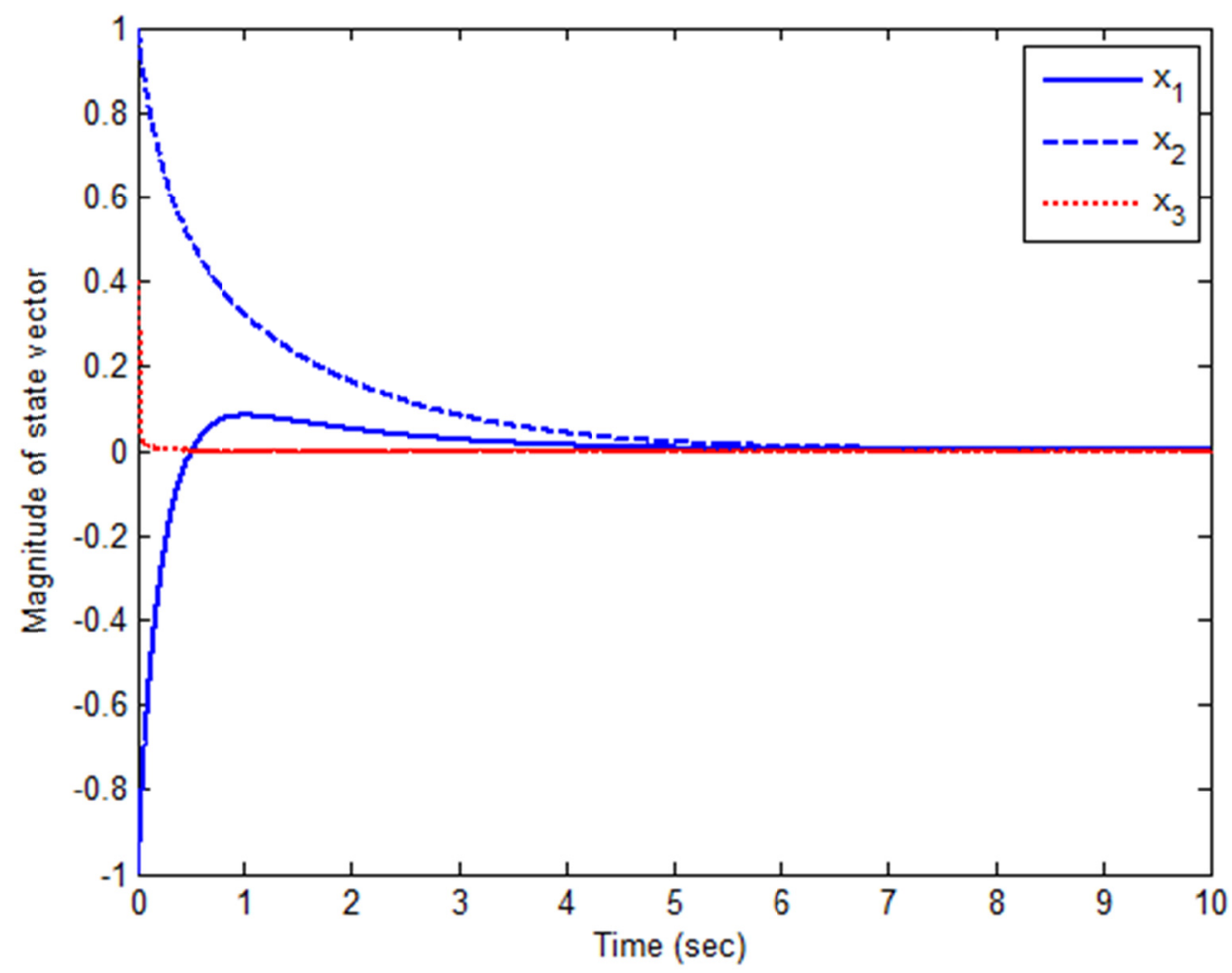

Figure 1. Time responses of system states $x(t)$ 


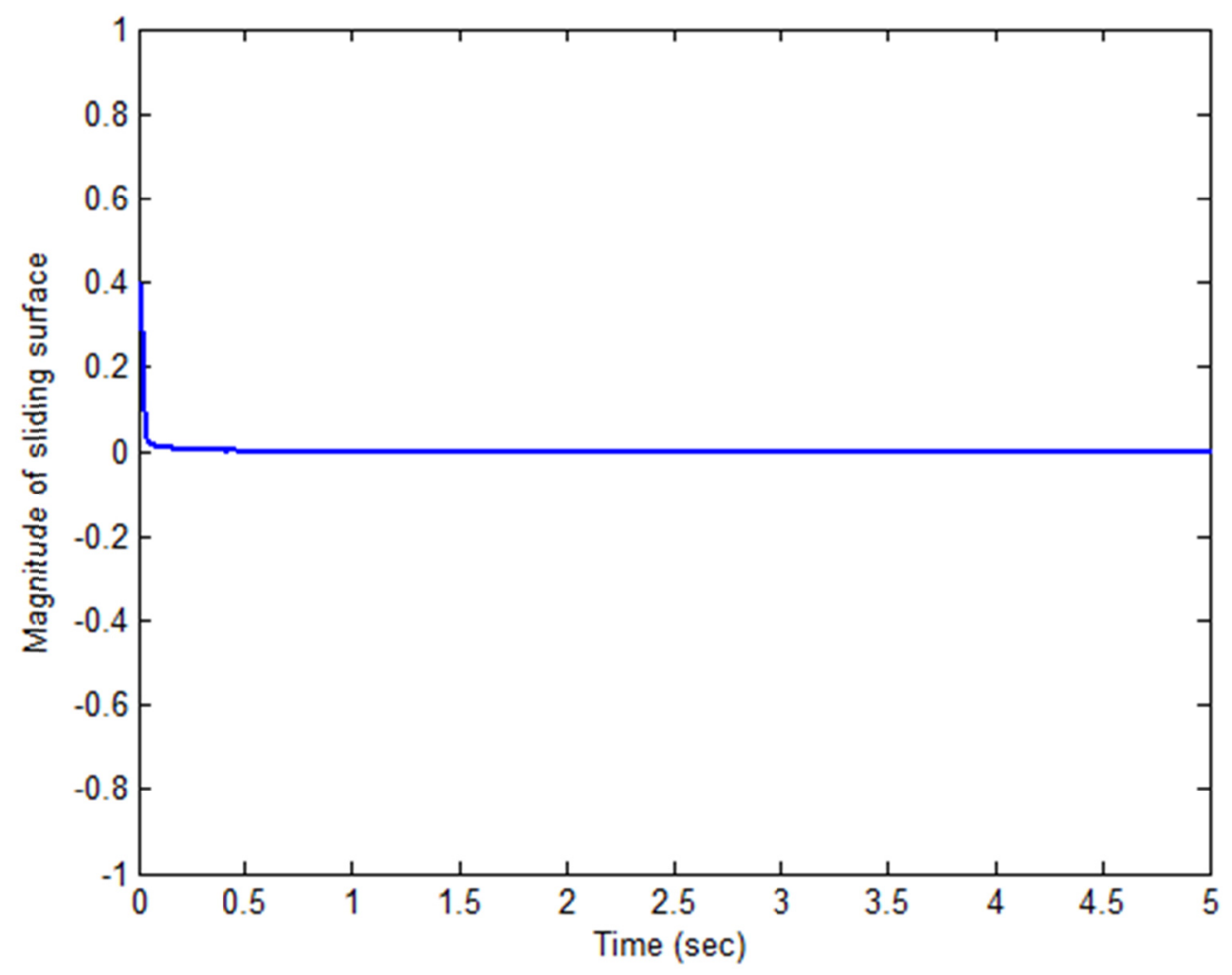

Figure 2. Time responses of the sliding surface $\sigma(t)$

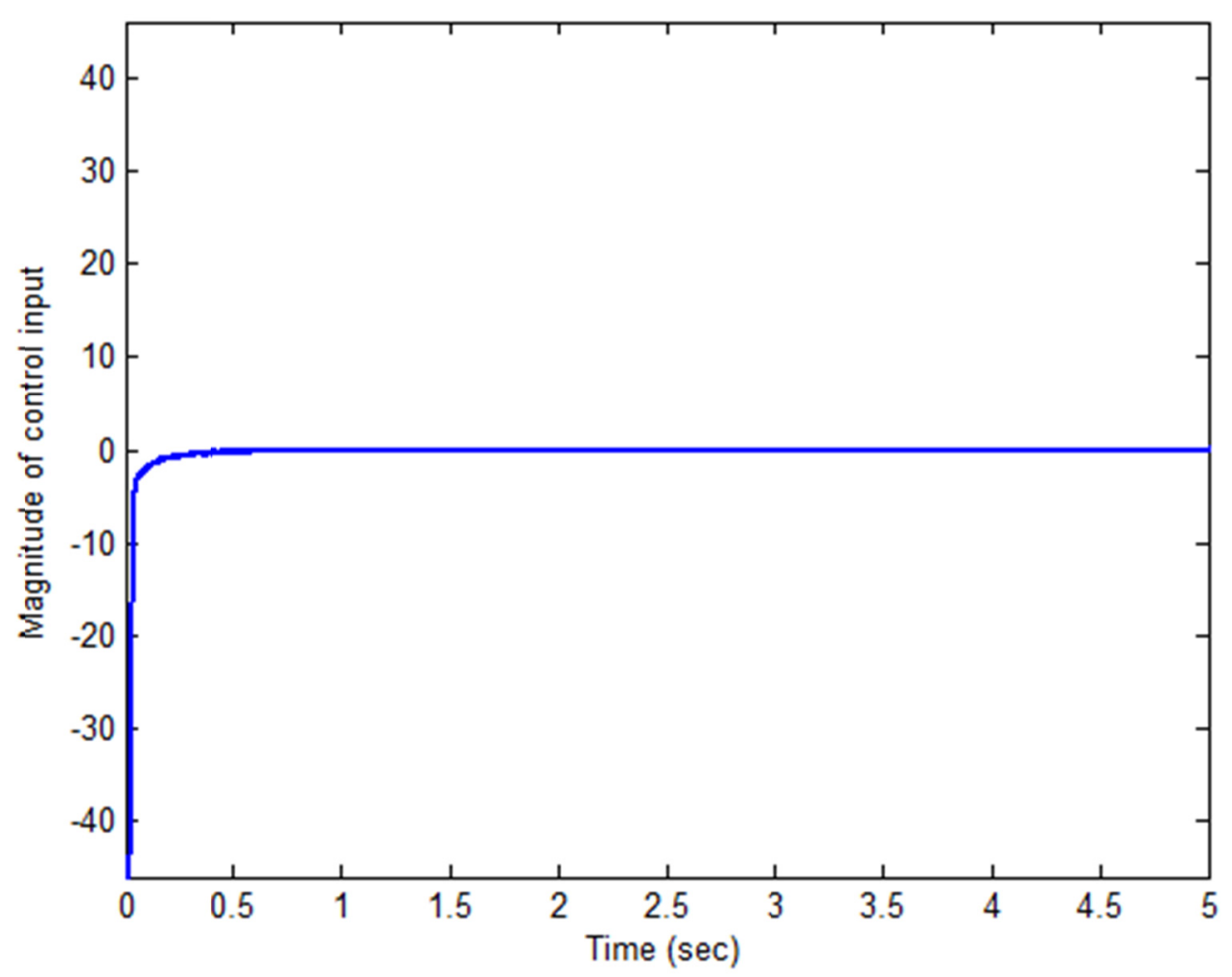

Figure 3. Time responses of the control input $u(t)$ 


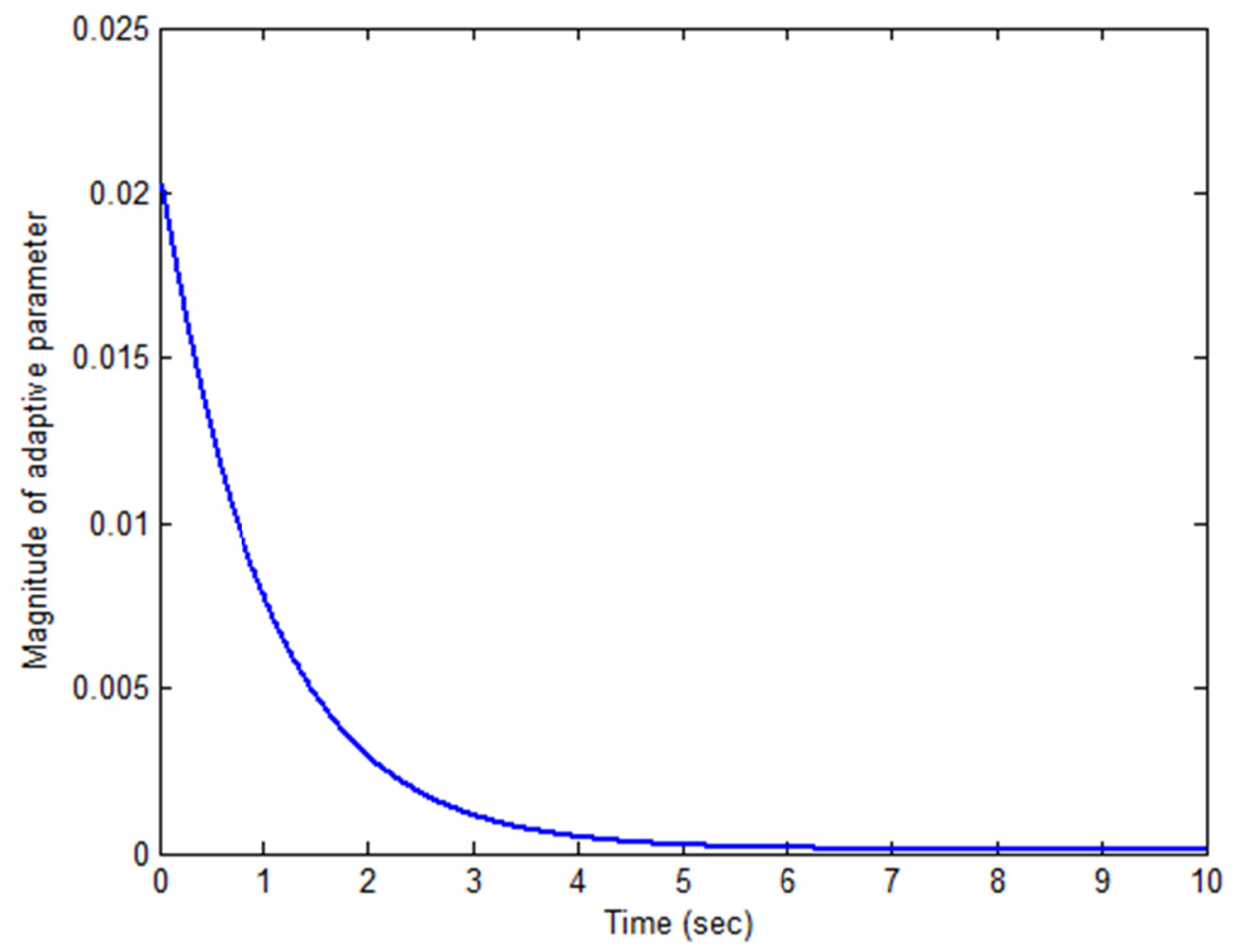

Figure 4. Time responses of the adaptive parameter $\hat{a}_{1}(t)$

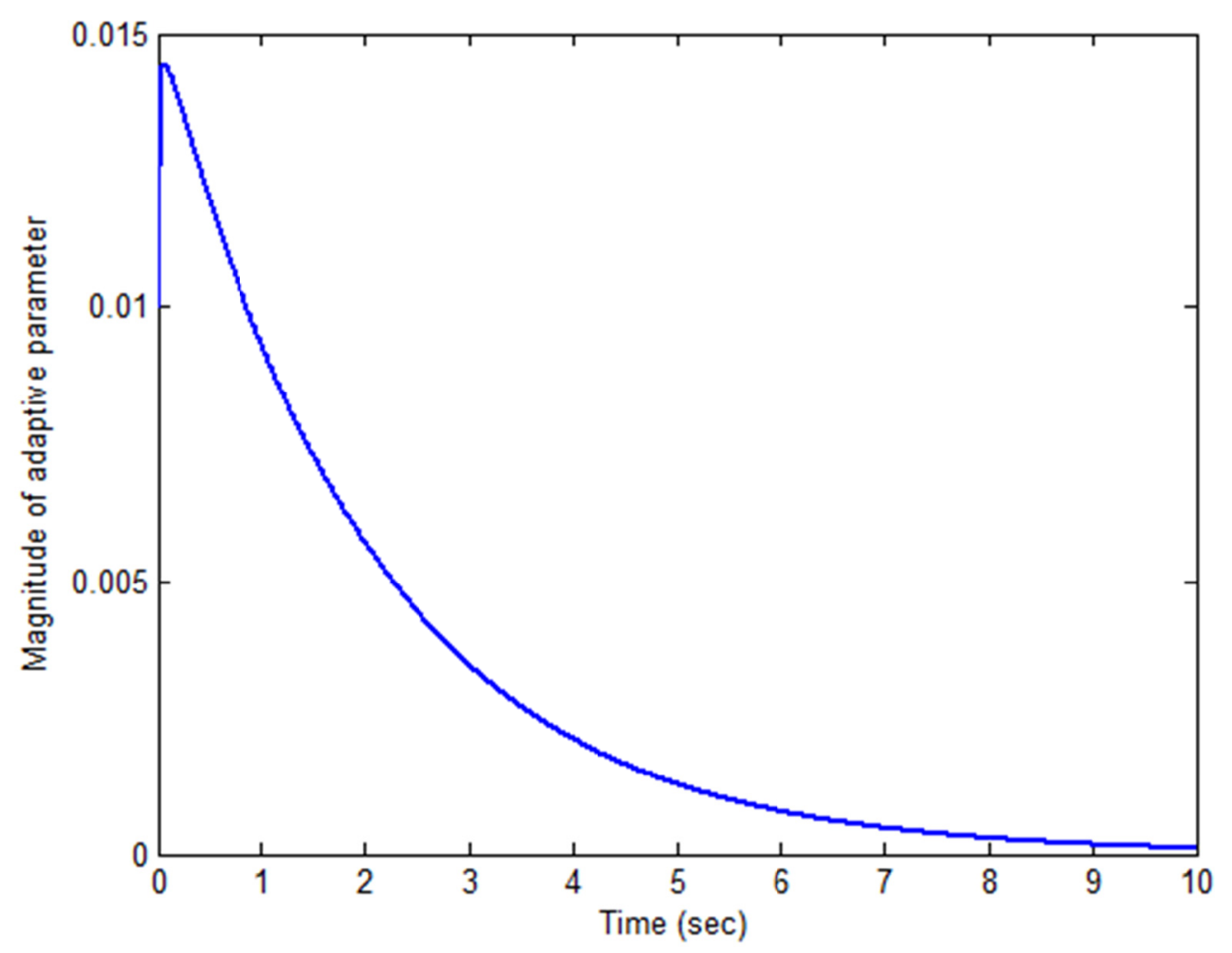

Figure 5. Time responses of the adaptive parameter $\hat{a}_{2}(t)$ 


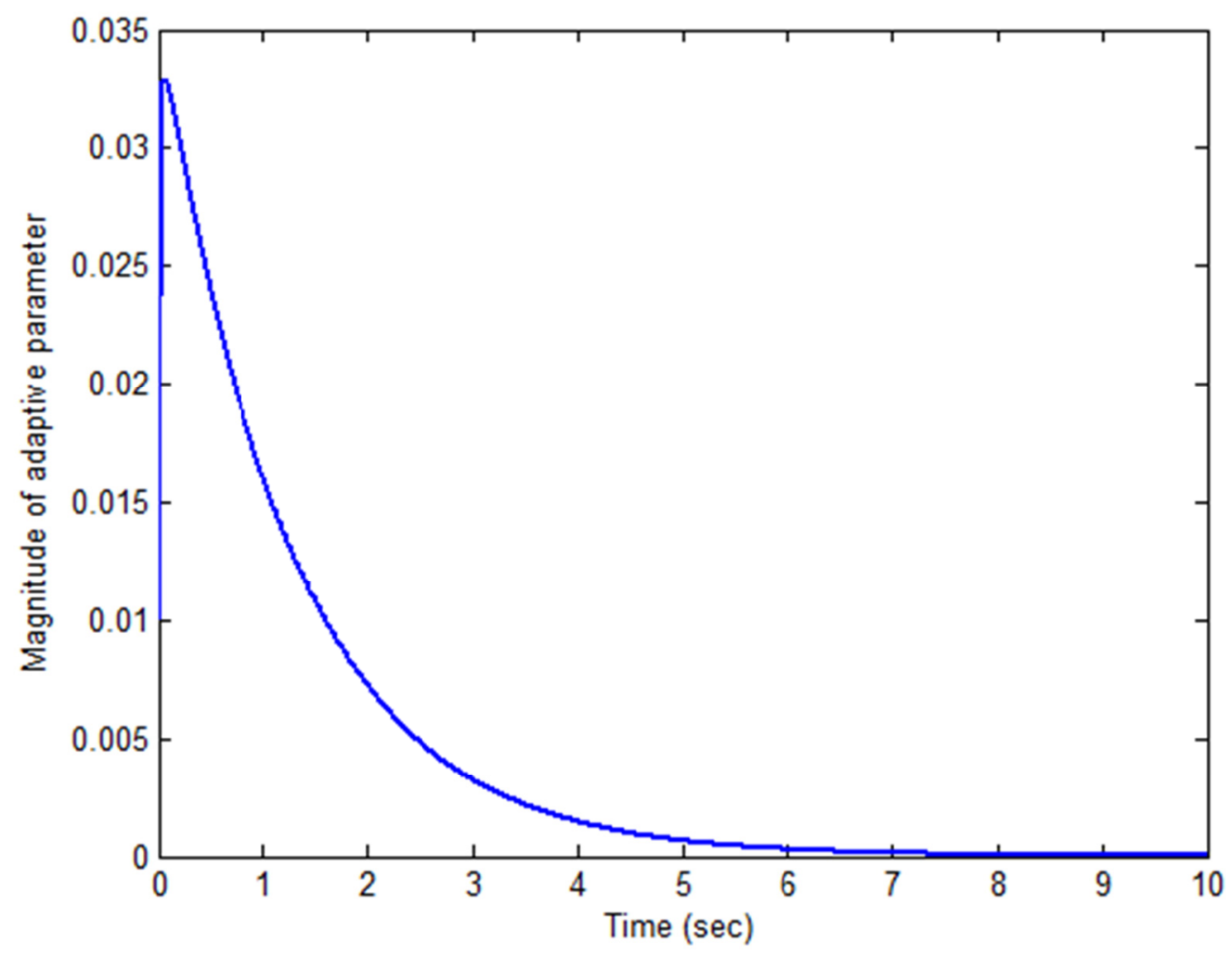

Figure 6. Time responses of the adaptive parameter $\hat{a}_{3}(t)$

The initial conditions for the above system are selected to be $x(0)=\left[\begin{array}{lll}-1 & 1 & 0.4\end{array}\right]^{T}$. The simulation results are shown in Figures 1 and 6. Figure 1 shows the trajectories of all the states which it is obtained that the asymptotic stability is achieved as proves by theorem 1. Figure 2 illustrates the sliding surface $\sigma(y, t)=0$ achieved at $t=0.2$ about and the effect of the uncertainties are effectively eliminated. Figure 3 shows the control input. It can be seen that the proposed controller has a good performance and is effective in dealing with matched and mismatched uncertainty system.

Remark 3: This approach uses only output information completely in sliding surface and controller design. Thus, conservatism is reduced and robustness is enhanced.

\section{Conclusion}

This paper has presented the adaptive variable structure control for mismatched uncertain systems where disturbances are bounded by an unknown function. In the sliding mode, asymptotic stability of the mismatched uncertain system is assured under certain conditions. Based on modified adaptive law, the adaptive output feedback variable structure control is designed to solve unknown disturbances and guarantee the reachability of system states. This approach does not need the availability of the state variables so that this method is very useful and more realistic since it can be easily implemented in many practical systems.

\section{References}

Choi, H. H. (1998). An explicit formula of linear sliding surfaces for a class of uncertain dynamic systems with mismatched uncertainties. Automatica, 34, 1015-1020. http://dx.doi.org/10.1016/S0005-1098(98)00042-9

Diong, M. B., \& Medanic, J. V. (1992). Dynamic output feedback variable structure control for system stabilization. International Journal of Control, 56(3), 607-630. http://dx.doi.org/10.1080/00207179208934331

Edwards, C., \& Spurgeon, S. K. (1998). Sliding mode control: theory and applications. Taylor and Francis Ltd, London.

Emelyanov, S. V., Korovinj, S. K., Nersisyan, A. L., \& Nisenzov, Y. E. (1990). Output feedback stabilization of uncertain plants: a variable structure systems approach. International Journal of Control, 55(1), 61-81. http://dx.doi.org/10.1080/00207179208934226

Esfandiari, F., \& Khalil, H. K. (1992). Output feedback stabilization of fully linearizable systems. International Journal of Control, 56(5), 1007-1037. http://dx.doi.org/10.1080/00207179208934355 
Ginoya, D., Shendge, P. D., \& Phadke, S. B. (2014). Sliding mode control for mismatched uncertain systems using an extended disturbance observer. IEEE Transactions on Industrial Electronics, 61, 1983-1992. http://dx.doi.org/10.1109/TIE.2013.2271597

Hao, L. Y., \& Yang, G. H. (2013). Fault-tolerant control via sliding-mode output feedback for uncertain linear systems with quantisation. IET Control Theory \& Applications, $7(16), \quad 1992-2006$. http://dx.doi.org/10.1049/iet-cta.2013.0380

Kwan, C. (2001). Further result on variable output feedback controllers. IEEE Transactions on Automatic Control, 46(9), 1505-1508. http://dx.doi.org/10.1109/9.948487

Kwan, C. M. (1996). On variable structure output feedback controllers. IEEE Transactions on Automation Control, 41, 1691-1693. http://dx.doi.org/10.1109/9.544012

Nunes, E. V. L., Hsu, L., \& Lizarralde, F. (2009). Global Exact Tracking for Uncertain Systems Using Output-Feedback Sliding Mode Control. IEEE Transactions on Automatic Control, 54(5), 1141-1147. http://dx.doi.org/10.1109/TAC.2009.2013042

Shyu, K. K., Tsai, Y. W., \& Lai, C. K. (2001). A dynamic output feedback controllers for mismatched uncertain variable structure systems. Automatica, 37, 775-779. http://dx.doi.org/10.1016/S0005-1098(01)00014-0

Silva, J. M. A. D., Edwards, C., \& Spurgeon, S. K. (2009). Sliding-Mode Output-Feedback Control Based on LMIs for Plants With Mismatched Uncertainties. IEEE Transactions on Industrial Electronics, 56(9), 3675-3683. http://dx.doi.org/10.1109/TIE.2009.2024094

Tsai, Y. W., Mai, K. H., \& Shyu, K. K. (2006). Sliding mode control for unmatched uncertain systems with totally invariant property and exponential stability. Journal of the Chinese Institute of Engineers, 29, 179-183. http://dx.doi.org/10.1080/02533839.2006.9671111

Yan, G. X., Spurgeon, S. K., \& Edwards (2012). C. Global decentralised static output feedback sliding mode control for interconnected time-delay systems. IET Control Theory \& Application, 6(2), 192-202.

Zak, S. H., \& Hui, S. (1993). On variable structure output feedback controllers for uncertain dynamic systems. IEEE Transactions on Automation Control, 38, 1509-1512. http://dx.doi.org/10.1109/9.241564

Zhang, J., \& Zheng, W. X. (2014). Design of adaptive sliding mode controllers for linear systems via output feedback. IEEE Transactions on Industrial Electronics, 64, 3553-3562. http://dx.doi.org/10.1109/TIE.2013.2281161

\section{$(\mathrm{oc}) \mathrm{EY}$}

This work is licensed under a Creative Commons Attribution 3.0 License. 